\title{
Suppression of Angular Forces in Collisions of Non- S -State Transition Metal Atoms
}

\section{Citation}

Krems, R. V., J. Kłos, M. F. Rode, M. M. Szcz\#śniak, G. Chatasiński, and A. Dalgarno. 2005. "Suppression of Angular Forces in Collisions of Non-S-State Transition Metal Atoms." Physical Review Letters 94 (1). https://doi.org/10.1103/physrevlett.94.013202.

\section{Permanent link}

http://nrs.harvard.edu/urn-3:HUL.InstRepos:41417300

\section{Terms of Use}

This article was downloaded from Harvard University's DASH repository, and is made available under the terms and conditions applicable to Other Posted Material, as set forth at http:// nrs.harvard.edu/urn-3:HUL.InstRepos:dash.current.terms-of-use\#LAA

\section{Share Your Story}

The Harvard community has made this article openly available.

Please share how this access benefits you. Submit a story.

Accessibility 


\title{
Suppression of Angular Forces in Collisions of Non-S-State Transition Metal Atoms
}

\author{
R. V. Krems, ${ }^{1,4}$ J. Kłos, ${ }^{2}$ M. F. Rode, ${ }^{2}$ M. M. Szczȩśniak, ${ }^{2}$ G. Chałasiński, ${ }^{2,3}$ and A. Dalgarno ${ }^{4}$ \\ ${ }^{1}$ Harvard-MIT Center for Ultracold Atoms, Department of Physics, Harvard University, Cambridge, Massachusetts 02138, USA \\ ${ }^{2}$ Department of Chemistry, Oakland University, Rochester, Michigan 48309, USA \\ ${ }^{3}$ Faculty of Chemistry, Warsaw University, Pasteura 1, 02-093 Warszawa, Poland \\ ${ }^{4}$ Institute for Theoretical Atomic, Molecular and Optical Physics, Harvard-Smithsonian Center for Astrophysics, \\ Cambridge, Massachusetts 02138, USA \\ (Received 10 June 2004; published 3 January 2005; corrected 5 January 2005)
}

\begin{abstract}
Angular momentum transfer is expected to occur rapidly in collisions of atoms in states of nonzero angular momenta due to the large torque of angular forces. We show that despite the presence of internal angular momenta transition metal atoms interact in collisions with helium effectively as spherical atoms and angular momentum transfer is slow. Thus, magnetic trapping and sympathetic cooling of transition metal atoms to ultracold temperatures should be readily achievable. Our results open up new avenues of research with a broad class of ultracold atoms.
\end{abstract}

DOI: 10.1103/PhysRevLett.94.013202

PACS numbers: $34.20 . \mathrm{Cf}, 32.60 .+\mathrm{i}, 32.80 . \mathrm{Pj}, 34.50 .-\mathrm{s}$

The realization of Bose-Einstein condensation (BEC) and the creation of Fermi degenerate gases of atoms have greatly expanded our understanding of nature. Novel phenomena such as BEC of a Fermi gas [1], vortices in atomic clouds [2], and retardation in atom-surface interactions [3] have been observed. External field control of atomic collisions has become possible [4]. The experiments on quantum degeneracy have been limited to ground-state alkali metal atoms, hydrogen and helium because BEC occurs at extremely low temperatures and, with few exceptions, the creation of ultracold atoms relies on evaporative cooling in a magnetic trap [5]. Evaporative cooling rests upon energy dissipation in elastic collisions between atoms, while the atoms must remain in the Zeeman state with the highest energy to stay trapped. Evaporative cooling has been applied only to atoms without internal orbital angular momentum - $S$-state atoms - for which Zeeman relaxation is inefficient. By contrast, the Zeeman relaxation in collisions of non-S-state atoms - atoms with internal orbital angular momenta - is induced by strong electrostatic and exchange interactions and it may be very fast [6]. Here we show that, despite the presence of orbital angular momenta, non- $S$-state transition metal atoms interact upon collisions with $\mathrm{He}$ atoms effectively like spherically symmetric $S$-state atoms. Our results suggest the possibility of magnetic trapping and sympathetic cooling of non- $S$-state atoms by collisions with trapped $S$-state atoms to ultracold temperatures and open up avenues for research with a new broad class of ultracold atoms.

The electronic interaction between non- $S$-state atoms depends strongly on the relative orientation of the vector separating the atoms and the orientation of the atomic orbitals with unpaired electrons [6,7]. The angular dependence of the interaction potential induces a large torque that spins up the angular momentum of the colliding atoms. In a magnetic field, such angular momentum rotation leads to Zeeman transitions. Previous theoretical work [8-10] showed that the probability of Zeeman relaxation in cold and ultracold collisions of open-shell $\mathrm{Ca}\left({ }^{3} P\right), \operatorname{Sr}\left({ }^{3} P\right)$ and $\mathrm{O}\left({ }^{3} P\right)$ atoms is very large, as expected. Less then ten collisions suffice to bring about angular momentum reorientation. Here we show that the interaction of non- $S$-state transition metal atoms $\operatorname{Sc}\left({ }^{2} D\right)$ and $\operatorname{Ti}\left({ }^{3} F\right)$ with groundstate He atoms is dominated by the spherically symmetric repulsive exchange interaction and that it suppresses the role of internal angular momentum in the collision process.

Our choice of ${ }^{3} \mathrm{He}$ as the collision partner for $\mathrm{Sc}$ and Ti is motivated by its use in buffer-gas loading experiments [11]. Buffer-gas loading is a general technique for magnetic trapping of atoms. Atoms are cooled by elastic collisions with $\mathrm{He}$ atoms and captured in a magnetic trap for which their translational energy is smaller than the trap depth. The Zeeman relaxation rates in collisions of Sc and Ti with ${ }^{3} \mathrm{He}$ have been recently measured [12]. He is an $S$-state atom so the angular dependence of the interaction of $\mathrm{Ti}$ and $\mathrm{Sc}$ with $\mathrm{He}$ is entirely determined by the electronic structure of the transition metal atoms.

The interaction potential between an atom in a state with nonzero electronic orbital angular momentum such as $\mathrm{Ti}\left({ }^{3} F\right)$ and $\mathrm{Sc}\left({ }^{2} D\right)$ and an $S$-state atom such as He can be written in the effective potential form $[13,14]$

$$
V=\sum_{\lambda} V_{\lambda}(R) P_{\lambda}
$$

where $R$ is the interatomic distance and $P_{\lambda}$ is the Legendre polynomial of order $\lambda$. The term with $\lambda=0$ is the isotropic part of the potential, while the terms with $\lambda>0$ represent the angular dependence of the interaction potential or the electronic interaction anisotropy. The expansion coefficients $V_{\lambda}(R)$ can be related to the nonrelativistic BornOppenheimer interaction potentials of the diatomic molecule $[6,7]$. The relations for $D$-state atoms have the form $[6,7]$ 


$$
\begin{aligned}
& V_{\lambda=0}=\left(V_{\Sigma}+2 V_{\Pi}+2 V_{\Delta}\right) / 5 \\
& V_{\lambda=2}=\left(V_{\Sigma}-V_{\Delta}\right)+\left(V_{\Pi}-V_{\Delta}\right) \\
& V_{\lambda=4}=9 / 5\left(V_{\Sigma}-V_{\Pi}\right)+3 / 5\left(V_{\Delta}-V_{\Pi}\right),
\end{aligned}
$$

and for $F$-state atoms, they are $[6,7]$

$$
\begin{aligned}
V_{\lambda=0}= & \left(V_{\Sigma}+2 V_{\Pi}+2 V_{\Delta}+2 V_{\Phi}\right) / 7 \\
V_{\lambda=2}= & 5\left(V_{\Sigma}-V_{\Phi}\right) / 7+15\left(V_{\Pi}-V_{\Phi}\right) / 14 \\
V_{\lambda=4}= & 9\left(V_{\Sigma}-V_{\Delta}\right) / 7+3\left(V_{\Pi}-V_{\Delta}\right) / 7+9\left(V_{\Phi}-V_{\Delta}\right) / 7 \\
V_{\lambda=6}= & 13\left(V_{\Sigma}-V_{\Pi}\right) / 7+13\left(V_{\Delta}-V_{\Pi}\right) / 14+13\left(V_{\Delta}\right. \\
& \left.-V_{\Phi}\right) / 70,
\end{aligned}
$$

where $V_{\Sigma}, V_{\Pi}, V_{\Delta}$, and $V_{\Phi}$ are the Born-Oppenheimer potentials of the $\mathrm{Sc}-\mathrm{He}$ or Ti-He molecules. Equations (2) and (3) establish that the electronic interaction anisotropy in Sc-He and Ti-He is determined by the splitting of the Born-Oppenheimer potentials with different symmetries. Similarly, the interaction anisotropy in complexes of $P$-state atoms with $\mathrm{He}$ is determined by the splitting between the potentials of $\Sigma$ and $\Pi$ symmetry [7].

The interaction of the $\mathrm{Sc}$ atom in the ground-state ${ }^{2} D$ with $\mathrm{He}$ gives rise to three adiabatic potentials: $V_{\Sigma}, V_{\Pi}$, and $V_{\Delta}$. We computed the interaction potentials of the ScHe complex using a single-reference spin unrestricted coupled cluster method with single, double, and noniterative triple excitations. The one-electron basis set consisted of the Bauschlicher's contracted set [15] for $\mathrm{Sc}$ and $\mathrm{Ti}$, and the augmented correlation-consistent polarized quadruplezeta set (aug-cc-pVQZ) [16] without the $g$-type atomic orbitals for He. To ensure a proper description of the dispersion interaction, we placed a set of bond functions $[3 s 3 p 2 d 1 g 1 h] \quad$ with exponents sp 0.9, 0.3,0.1; $d f 0.6,0.2 ; g h 0.3$ in the middle of the van der Waals bond. The $1 s$ orbital of Sc was frozen in this calculation. We found that freezing the lowest five orbitals $\left(1 s, 2 s, 2 p_{x}\right.$, $2 p_{y}$, and $2 p_{z}$ ) of the metal did not change the results.

The interaction of $\left.\operatorname{Ti}^{3} F\right)$ with $\mathrm{He}$ gives rise to four adiabatic potentials: $V_{\Sigma}, V_{\Pi}, V_{\Delta}$, and $V_{\Phi}$. The singlereference approach is not appropriate in this case and we used a state-average complete active space self-consistent field (CASSCF) approach [17] in conjunction with the internally contracted multi-reference configuration interaction (MRCI) method [18] to compute these potentials. The basis set for the Ti-He calculations was analogous to that in the $\mathrm{Sc}-\mathrm{He}$ calculation but no bond functions were included in the MRCI calculations.

The interaction potentials $V_{i}(R)(i=\Sigma, \Pi, \Delta, \ldots)$ were obtained using the counterpoise correction procedure

$$
\begin{aligned}
V_{i}(R)= & E_{\mathrm{Me}-\mathrm{He}}^{i}(R ; \mathrm{DBS})-E_{\mathrm{Me}}^{i}(R ; \mathrm{DBS}) \\
& -E_{\mathrm{He}}(R ; \mathrm{DBS})-\Delta E_{\mathrm{SC}}^{i}
\end{aligned}
$$

individually applied to each state. DBS stands for the dimer basis set, Me denotes the metal atom and $\Delta E_{\mathrm{SC}}^{i}$ is a residual size-consistency correction. The last term is not necessary in the coupled cluster single and doubles with perturbative triples $[\mathrm{CCSD}(\mathrm{T})]$ calculations. Note that the degeneracy of the atomic states is removed in the full dimer basis set. In the MRCI calculations, the effect of higher excitations was estimated using the Pople size-consistency corrections [19]. The $\Delta E_{\mathrm{SC}}^{i}$ term was obtained by subtracting the dimer and monomer energies in Eq. (4) evaluated at $R=24 \AA$. The calculations were carried out with the MOLPRO suite of programs [20]. The CASSCF active space included five $d$ orbitals of $\mathrm{Me}$ and $1 s$ orbital of He with the lowest five orbitals frozen. A state averaging with equal weights was applied to all the states. More details of these calculations will be published elsewhere [21]. The calculations of the $\mathrm{O}-\mathrm{He}$ interaction potential, which is included for comparison, were carried out at the restricted $\operatorname{CCSD}(\mathrm{T})$ level of theory in the aug-cc-pVQZ basis set augmented by the bond functions described above.

Figs. 1 and 2 show the Born-Oppenheimer potentials for Sc-He and Ti-He in the vicinity of the van der Waals well and at long range. Two features are striking: the van der Waals minima are very shallow and the potentials of different symmetries are nearly degenerate at all interatomic distances. That the splitting between the potentials is very small indicates suppression of the interaction anisotropy.

The ratio of the rate constants for elastic collisions and Zeeman relaxation is a quantitative measure of the interaction anisotropy in collision complexes. We computed the elastic to inelastic ratios (see Table I) using a quantum mechanical method presented earlier $[8,22]$. The scattering

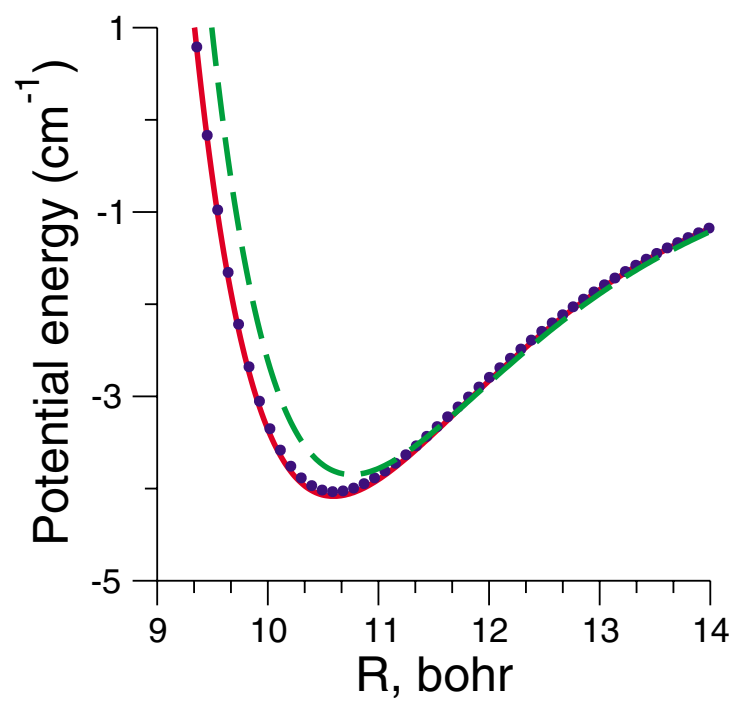

FIG. 1 (color online). $\mathrm{Sc}\left({ }^{2} D\right)$-He interaction potentials from $\operatorname{CCSD}(\mathrm{T})$ calculations: full line $-\Sigma$ symmetry; dashed line $-\Delta$ symmetry; symbols- $-\Pi$ symmetry. 


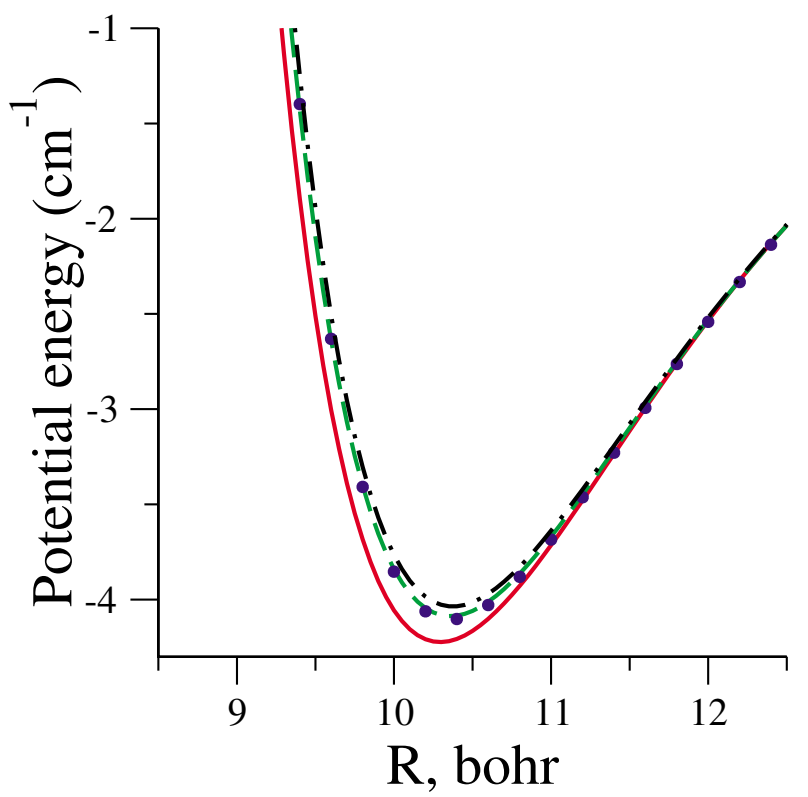

FIG. 2 (color online). $\quad \mathrm{Ti}\left({ }^{3} F\right)$-He interaction potentials from MRCI calculations: full line $-\Sigma$ symmetry; dashed line $-\Pi$ symmetry; dot-dashed line- $\Phi$ symmetry; symbols $-\Delta$ symmetry.

calculations were performed for a magnetic field strength of 3.8 Tesla and the rate constants were computed for the temperature $T=1.8 \mathrm{~K}$ for comparison with recent experiments [12]. The results are given in Table I. The elastic to inelastic ratio in $\mathrm{Sc}\left({ }^{2} D\right)$-He collisions is about 200 times larger than that in $\mathrm{O}\left({ }^{3} P\right)-\mathrm{He}$ collisions [8], and the rate of inelastic transitions in $\left.\operatorname{Ti}^{3} F\right)$-He collisions is reduced by almost 4 orders of magnitude. In agreement with measurements [12] we find large values for the elastic to inelastic ratio in Ti-He and Sc-He collisions. The calculations show that the anisotropy in the Ti-He interaction is smaller than in the Sc-He interaction.

We argue that the suppression of the interaction anisotropy in the Ti-He and $\mathrm{Sc}-\mathrm{He}$ complexes is due to the specific structure of the transition metal atoms and not due to the $D$ or $F$ character of the atomic terms. To support the argument we computed the interaction potential of helium with oxygen in the higher orbital momentum ${ }^{1} D$ state and with doubly ionized titanium $\mathrm{Ti}^{2+}\left({ }^{3} F\right)$. The

TABLE I. Ratio $\gamma$ of rate constants for elastic and inelastic collisions at $T=1.8 \mathrm{~K}$ and $B=3.8 \mathrm{~T}$.

\begin{tabular}{lcc}
\hline \hline Complex & $\gamma$ & $\gamma_{\exp }{ }^{\mathrm{a}}$ \\
\hline $\mathrm{O}\left({ }^{3} P\right)-{ }^{3} \mathrm{He}$ & 3 & \\
$\mathrm{O}\left({ }^{1} D\right)-\mathrm{He}$ & 1.6 & $<(1.6 \pm 0.3) \times 10^{4}$ \\
$\mathrm{Sc}\left({ }^{2} D\right)-{ }^{3} \mathrm{He}$ & 790 & $\sim(4.0 \pm 1.8) \times 10^{4}$ \\
$\mathrm{Ti}\left({ }^{3} F\right)-{ }^{3} \mathrm{He}$ & 6953 & \\
\hline \hline
\end{tabular}

${ }^{\mathrm{a}}$ The experimental value of $\gamma$ from Ref. [12]. potential curves are presented in Figs. 3 and 4. The scattering calculations in Table I of the elastic to inelastic ratio show that the interaction anisotropy in the $\mathrm{O}\left({ }^{1} D\right)$-He complex is comparable to that in the $\mathrm{O}\left({ }^{3} P\right)-\mathrm{He}$ complex, whereas the removal of two electrons from Ti, changing the electronic configuration of the atom from $3 d^{2} 4 s^{2}$ to $3 d^{2}$ [23], increases the anisotropy with $\mathrm{He}$ by 3 orders of magnitude.

The suppression of the anisotropy of the transition metal-He potentials can be attributed to the role of the repulsive exchange interaction. The average radius of the $4 s$ orbital in transition metal atoms is over two times larger than that of the $3 d$ orbital [24]. The ratio of the radial extent of the $4 s$ to $3 d$ orbitals varies from 2.0 to 3.4 in the $\mathrm{Sc}-\mathrm{Cu}$ series [24] due to $d$-orbital collapse [25]. The exchange interaction due to the overlap of electronic functions of the colliding atoms thus involves primarily the $1 s$ orbital of $\mathrm{He}$ and the $4 s$ orbital of the transition metal atoms. The overlap leads to an isotropic exchange repulsion resulting in nearly degenerate energies of the $\Sigma, \Pi, \Delta$, and $\Phi$ states and to very shallow long-range van der Waals minima. Removal of the outer electrons from the $4 s$ orbital exposes the $3 d$ orbitals to a substantial overlap with the $1 s \mathrm{He}$ orbital which leads to the strongly anisotropic interaction shown in Fig. 4.

In summary, we have demonstrated by explicit quantum mechanical calculations that the electronic interaction anisotropies in complexes of transition metal atoms $\mathrm{Sc}$ and $\mathrm{Ti}$ with $\mathrm{He}$ are dramatically suppressed. As a result of the $d$-orbital collapse in transition metal atoms, the interaction between $\mathrm{Sc}$ or $\mathrm{Ti}$ and $\mathrm{He}$ is dominated by the repulsive

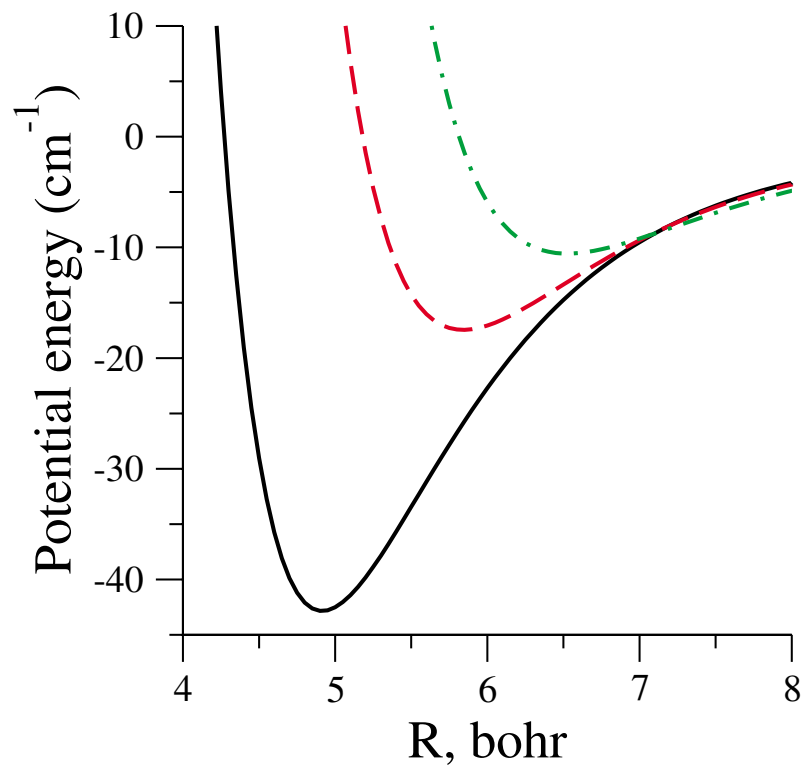

FIG. 3 (color online). $\mathrm{O}\left({ }^{1} D\right)$-He interaction potentials from $\operatorname{CCSD}(\mathrm{T})$ calculations: full line $-\Sigma$ symmetry; dashed line$\Pi$ symmetry; dot-dashed line $-\Delta$ symmetry. 


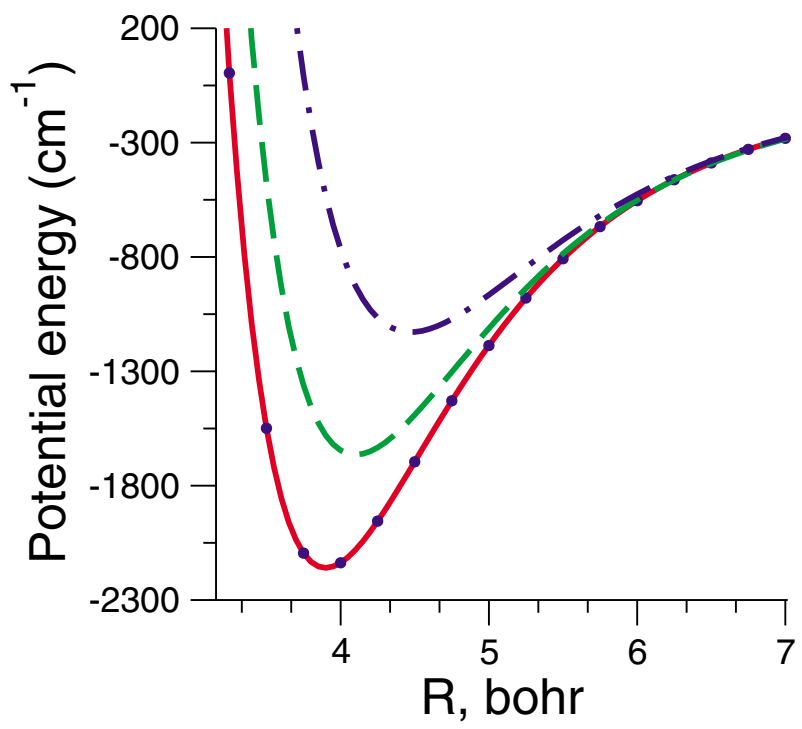

FIG. 4 (color online). $\quad \mathrm{Ti}^{2+}\left({ }^{3} F\right)$-He interaction potentials from MRCI calculations: full line $-\Sigma$ symmetry; dashed line $-\Pi$ symmetry; dot-dashed line $-\Delta$ symmetry; symbols $-\Phi$ symmetry.

exchange interaction which is found to be spherically symmetric. The probability of the angular momentum transfer in collisions of transition metal atoms with spherically symmetric atoms is very low. This suggests that transition metal atoms may be cooled and magnetically trapped in a buffer gas of He. Interactions of transition metal atoms with $S$-state alkali atoms in a magnetic trap will be characterized by similar features as observed in this Letter [6]. Our results suggest that transition metal atoms can be cooled to ultracold temperatures sympathetically by elastic collisions with trapped alkali metal atoms. The magnetic dipole moment of many transition metal atoms is small - in Sc it is 1.2 and in Ti, 1.3 Bohr magnetons - so the magnetic dipole interactions will be ineffective in collisions with alkali metal atoms.

Ultralow temperature studies of $S$-state alkali metal atoms have revolutionized atomic and molecular physics in the past decade. Most of the atoms in the periodic table are non- $S$-state atoms and possibilities of new discoveries with ultracold non- $S$-state atoms are boundless. They include new methods to study fundamental symmetries, new systems to search for the electric dipole moment of the electron and the time variation of the fine structure constant, new constituents for quantum computation, and novel atom-molecule reaction mechanisms at very low temperatures. Our work is a critical step in the ultracold physics of non- $S$-state atoms.

M.M.S. and G.C. are supported by the National Science Foundation (Grant No. CHE-0414241), and R. V.K. and A.D. are supported by the National Science Foundation through grants to ITAMP and CUA and a grant from Chemical Sciences, Geosciences and Biosciences
Division of the Office of Basic Energy Sciences, Office of Science, U. S. Department of Energy.

[1] M. Greiner, C. A. Regal, and D. S. Jin, Nature (London) 426, 537 (2003).

[2] J. R. Anglin and W. Ketterle, Nature (London) 416, 211 (2002).

[3] Yu-ju Lin, I. Teper, C. Chin, and V. Vuletic, Phys. Rev. Lett. 92, 050404 (2002).

[4] J. Stenger, S. Inouye, M. R. Andrews, H. J. Miesner, D. M. Stamper-Kurn, and W. Ketterle, Phys. Rev. Lett. 82, 2422 (1999).

[5] A.L. Migdall, J. V. Prodan, W.D. Phillips, T.H. Bergeman, and H. J. Metcalf, Phys. Rev. Lett. 54, 2596 (1985).

[6] R. V. Krems, G. C. Groenenboom, and A. Dalgarno, J. Phys. Chem. A 108, 8941 (2004).

[7] V. Aquilanti and G. Grossi, J. Chem. Phys. 73, 1165 (1980).

[8] R. V. Krems and A. Dalgarno, Phys. Rev. A 68, 013406 (2003).

[9] V. Kokoouline, R. Santra, and C. H. Greene, Phys. Rev. Lett. 90, 253201 (2003).

[10] R. V. Krems, Phys. Rev. Lett. 93, 013201 (2004).

[11] J. Weinstein, R. deCarvalho, T. Guillet, B. Friedrich, and J. M. Doyle, Nature (London) 395, 148 (1998).

[12] C.I. Hancox, S. C. Doret, M. T. Hummon, R. V. Krems, and J. M. Doyle, this issue, Phys. Rev. Lett. 94, 013201 (2005).

[13] J. Callaway, and E. Bauer, Phys. Rev. 140, A1072 (1965).

[14] R. H. G. Reid and A. Dalgarno, Phys. Rev. Lett. 22, 1029 (1969).

[15] C. W. Bauschlicher, Jr., Theor. Chim. Acta 92, 183 (1995).

[16] D. E. Woon and T.H. Dunning, Jr., J. Chem. Phys. 100, 2975 (1994).

[17] H.-J. Werner and W. Meyer, J. Chem. Phys. 74, 5794 (1981).

[18] H.-J. Werner and P. J. Knowles, J. Chem. Phys. 89, 5803 (1988).

[19] J. A. Pople, J. S. Binkley, and R. Seeger, Int. J. Quantum Chem. S11, 149 (1977).

[20] MOLPRO is a package of ab initio programs written by H.J. Werner and P. J. Knowles, with contributions from R. D. Amos, A. Berning, D. L. Cooper, M. J. O. Deegan, A.J. Dobbyn, F. Eckert, C. Hampel, T. Leininger, R. Lindh, A. W. Lloyd, W. Meyer, M.E. Mura, A. Nicklaß, P.Palmieri, K. Peterson, R. Pitzer, P. Pulay, G. Rauhut, M. Schütz, H. Stoll, A. J. Stone, and T. Thorsteinsson.

[21] J. Kłos, M. F. Rode, J. E. Rode, G. Chałasiński, and M. M. Szczęśniak, Eur. Phys. J. D 31, 429 (2004).

[22] R. V. Krems and A. Dalgarno, J. Chem. Phys. 120, 2296 (2004).

[23] See the NIST atomic spectra database at http://physics.nist.gov/cgi-bin/AtData/main asd.

[24] S. R. Langhoff and C. W. Bauschlicher, Jr., Annu. Rev. Phys. Chem. 39, 181 (1988).

[25] D. C. Griffin, K. L. Andrew, and R. D. Cowan, Phys. Rev. 177, 62 (1969). 